sion: tissue stores become partially depleted. J Lab Clin Med 138:206-213

13. Iskandar SB, Olive KE (2004) Plasmapheresis as an adjuvant therapy for hypertriglyceridemia-induced pancreatitis. Am J Med Sci 328:290-294

14. Malmström R, Packard CJ, Watson TDG, Rannikko S, Caslake M, Bedford D, Stewart P, Yki-Järvinen H, Shepherd H, Taskinen M-R (1997) Metabolic basis of hypotriglyceridemic effects of insulin in normal men. Arterioscler Thromb Vasc Biol 17:1454-1464

Intern Emerg Med (2007) 2:70-71

DOI 10.1007/s11739-007-0020-3

\section{D-dimer testing: advantages and limitations in emergency medicine for managing acute venous thromboembolism}

\section{Imberti}

\author{
D. Imberti (凶) \\ Thrombosis Center \\ Emergency Department \\ Ospedale Civile \\ Via Taverna 49, I-29100 Piacenza, Italy \\ e-mail: d.imberti@ausl.pc.it
}

Received: 5 October 2006 / Accepted in original form: 6 October 2006 / Published online: 31 March 2007

Patients with suspected deep vein thrombosis (DVT) or pulmonary embolism (PE) are frequently admitted to an Emergency Department (ED) for initial evaluation. However, management of patients with suspicion of acute venous thromboembolism (VTE) in this clinical setting can be difficult; in fact symptoms and signs of DVT are non-specific and can be found in a broad spectrum of non-thrombotic disorders. An accurate and timely objective diagnosis is necessary for immediate and correct identification of patients with acute VTE, while avoiding the bleeding risk associated with unnecessary anticoagulant therapy in patients where DVT or PE have been ruled out. The diagnostic approach to patients with suspected VTE includes clinical evaluation, diagnostic imaging and D-dimer testing [1,2].

In a recent issue of Internal and Emergency Medicine, Siragusa [3] exhaustively discussed the currently used assays, clinical indications and limitations of D-dimer testing for managing acute VTE in emergency medicine. Measurement of D-dimer values, a degradation product of cross-linked fibrin, is a simple laboratory test, and has been extensively studied in numerous prospective cohort studies in cases of suspected DVT or PE, showing a high negative predictive value. The author [3] shows that D-dimer testing has sufficient diagnostic accuracy for ruling out acute VTE if used in combination with standardised clinical judgement. The review is important for at least three reasons.

First, many physiological and pathological conditions can increase plasma D-dimer levels (pregnancy, age, trauma, cancer, inflammation and several other clinical conditions); on the other hand D-dimer levels may fail to increase in patients with acute VTE for multiple reasons (impaired fibrinolytic activity, use of heparin or oral anticoagulants, onset of symptoms more than two weeks before blood sampling). For these reasons, D-dimer testing has a high sensitivity but a low specificity in the diagnosis of acute VTE; in clinical practice it may be associated with frequent "false positive" results, but also more rarely with "false negative" findings. However, its use should always be associated with a careful clinical assessment to rule out the presence of acute VTE only in symptomatic patients. Overuse or misuse of the D-dimer screen for VTE may have negative consequences, in terms of a burden both for patients and for healthcare costs; in fact, despite clinical guidelines, inappropriate and unnecessary measurement of D-dimer is a significant clinical problem [4].

Second, commercially available assays are very different for many reasons. Assays differ in the type of antibody used as well as in the type of data provided, as some are qualitative whereas others are quantitative. Moreover, the value of various D-dimer assays is based upon several factors including sensitivity, specificity, cost, time required, reproducibility and complexity. Finally, the diagnostic accuracy of D-dimer relies on the cut-off values, that is the range within which the test has the best sensitivity and specificity, and may be very different comparing one assay to another. Cut-off points are established by the manufacturers, according to the characteristics of D-dimer testing; a wide range of sensitivities and specificities have been reported for different D-dimer assays. Currently, no Ddimer reference standard exists, making interassay correlation poor; moreover, because of variations and differences among different assays, results from one of them cannot be extrapolated to another.

Third, another interesting issue concerning the use of the D-dimer test in emergency medicine is its use when urgent diagnostic imaging for DVT or PE is not immediately available, as for example during a night-time or week-end evaluation. Siragusa and coworkers [5] studied whether a combined approach consisting of pre-test clinical probability, D-dimer testing and low-molecular-weight heparin (LMWH) allowed deferral of objective assessment of DVT or PE for up to $72 \mathrm{~h}$ in 409 patients admitted to ED for suspected VTE. Pending diagnostic confirmation, patients identified with a high probability or with a moderate probability and positive D-dimer test received a protective full dose of LMWH, while the remaining patients were discharged without anticoagulant treatment. The study demonstrates the safety of this approach.

Despite the promise of these findings, there are several points of caution worth noting with respect to the use 
of the D-dimer test in the ED. Researchers remain concerned that qualitative D-dimer assays may demonstrate lower diagnostic sensitivity compared with quantitative Ddimer assays, which are becoming more widely used in EDs to rule out the diagnosis of PE. Recently two important studies clearly demonstrated that a simplified D-dimer assay has a moderate sensitivity for PE in patients with clinical pretest low-risk probability [6] or is not sufficiently sensitive to exclude the diagnosis of PE in all symptomatic patients presenting to the ED with pleuritic chest pain [7]. In addition, as is reported by Squizzato and Ageno [8], physicians working in the ED should be adequately educated about the optimal use of D-dimer testing in diagnosing acute VTE. In fact, inappropriate use of D-dimer testing is a common error, and may cause several clinical problems; for example, given the high likelihood of false positive results, the costly and timeconsuming need for unnecessary additional imaging tests. Thus, it is mandatory that clinicians be aware of the potential limitations of D-dimer testing. It is clear for example that D-dimer testing has sufficient diagnostic accuracy for ruling out acute VTE only if it is used in combination with a standardised clinical probability score judgement, but not when used alone.

In conclusion, as Siragusa et al. report, I believe that Ddimer testing can be safely used in ED for ruling out acute VTE only if used in combination with a correct and validated pre-test clinical probability [5]. The reliance upon a Ddimer test seems to be a safe and reliable practice in the management of patients with suspicion of VTE when appropriate imaging tests are not immediately available. Moreover, because of its heterogeneity related to the method used, a Ddimer test accuracy should be carefully assessed in each hospital before its implementation in the emergency diagnosis of VTE. Finally, emergency physicians should receive adequate education concerning the correct use and limitations of a Ddimer test, to avoid error in its use in clinical practice.

\section{References}

1. Wells PS, Anderson DR, Rodger M et al (2003) Evaluation of DDimer in the diagnosis of suspected deep-vein thrombosis. $\mathrm{N}$ Engl J Med 349:1227-1235

2. Van Belle AF, Buller HR, Huisman MV et al for the Cristopher Study Investigators (2006) Effectiveness of managing suspected pulmonary embolism using an algorithm combining clinical probability, D-dimer testing, and computed tomography. JAMA 295:172-179

3. Siragusa $S$ (2006) D-dimer testing: advantages and limitations in emergency medicine for managing acute venous thromboembolism. Intern Emerg Med 1:59-66

4. Manfredini R (2006) D-dimer for the diagnosis of acute venous thromboembolism in the emergency department: a Janus-face marker. Intern Emerg Med 1:54-58

5. Siragusa S, Anastasio R, Porta C et al (2004) Deferment of objective assessment of deep vein thrombosis and pulmonary embolism without increased risk of thrombosis. A practical approach based on pre-test probability, D-Dimer testing, and the use of low-molecular weight heparins. Arch Intern Med 164:2477-2482

6. Kline JA, Runyon MS, Webb WB et al (2006) Prospective study of the diagnostic accuracy of the Simplify D-dimer assay for pulmonary embolism in emergency department patients. Chest 129:1417-1423

7. Hogg K, Dawson D, Mackway-Jones K (2005) The emergency department utility of Simplify D-dimer to exclude pulmonary embolism in patients with pleuritic chest pain. Ann Emerg Med 46:305-310

8. Squizzato A, Ageno W (2006) What is the next step in Ddimer research? Education of physicians [letter]. Intern Emerg Med 1:165 